\title{
Does Analog Transmission Achieve OPTA in an Asymmetric Gaussian Sensor Network ?*
}

\author{
Hamid Behroozi, Fady Alajaji and Tamás Linder \\ Department of Mathematics and Statistics, Queen's University, Kingston, Ontario, Canada, K7L 3N6 \\ Email: \{behroozi, fady, linder\}@ mast.queensu.ca
}

\begin{abstract}
We consider the estimation of a Gaussian source by a Gaussian sensor network where $L$ distributed sensors transmit noisy observations of the source through a Gaussian multiple access channel to a fusion center. In a recent work, Gastpar showed that for a symmetric sensor network with no fading, analog (uncoded) transmission achieves the optimal performance theoretically attainable (OPTA). In this work, by comparing lower and upper bounds on the OPTA, we provide optimality conditions for analog transmission in an asymmetric Gaussian sensor network with deterministic fading. We also obtain an optimal power allocation scheme to minimize the mean-squared error distortion given a linear combination of powers (LCP) constraint. We determine optimality conditions for analog transmission under an LCP constraint, which includes the sum-power constraint as a special case.
\end{abstract}

Index Terms - Power-distortion tradeoff, Gaussian multiple access channel, analog (or uncoded) transmission, sensor networks, linear combination of powers constraint, sum-power constraint.

\section{INTRODUCTION}

For the point-to-point transmission of a single Gaussian source through an additive white Gaussian noise (AWGN) channel it is known (e.g., see [1], [2]) that if the source and channel bandwidths are equal, simple analog transmission achieves the optimal performance theoretically attainable (OPTA). Analog (or uncoded) transmission in this case and in the rest of this paper means scaling the encoder input subject to the channel power constraint and transmitting it without explicit channel coding.

In this work, similar to [3]-[9], we consider the estimation of a memoryless Gaussian source by a Gaussian sensor network in which $L$ sensors observe the source signal $X$ corrupted by additive independent noise. The overall system is depicted in Fig. 1. The sensors communicate information about their observations through a Gaussian multiple access channel (MAC) with deterministic fading to a single fusion center (FC). According to [3], [4], [6], [9], the wireless links between sensors and the FC can be modeled as an AWN MAC with suitable channel gains. The encoders are distributed and cannot cooperate to exploit the correlation between their inputs. Each encoder is subject to a transmission cost constraint. The FC aims to reconstruct the main source, $X$, with the lowest mean squared-error (MSE) distortion at the smallest cost in the communication link. Note that the separate source and channel coding theorem of Shannon [10] does not hold in this problem [3], [7].

In [3], [7], [8], it is proved that analog transmission is exactly optimal for symmetric Gaussian sensor networks with

\footnotetext{
* This work was supported in part by a Postdoctoral Fellowship from the Ontario Ministry of Research and Innovation (MRI) and by the Natural Sciences and Engineering Research Council (NSERC) of Canada.
}

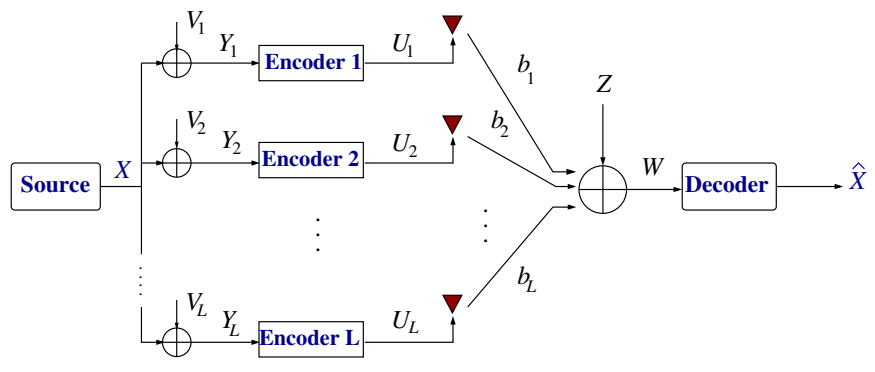

Fig. 1. A Gaussian sensor network with deterministic fading.

no fading. In the case of deterministic fading, lower and upper bounds on the minimum distortion are shown in [3], [5], and for random fading, bounds are also studied in [5], [11]. The minimum achievable distortion under a sum-power constraint for the analog transmission in sensor networks with deterministic fading is presented in [6]. The optimality of analog transmission in some other multi-user communication systems was recently shown in [4], [12].

Our main contribution in this paper is to provide optimality conditions for analog transmission under a linear combination of powers (LCP) constraint. Aside from being a natural generalization of the sum-power constraint, the LCP constraint explicitly allows to introduce weight coefficients that reflect the potentially differing costs of supplying power to individual sensors.

First, by comparing known lower and upper bounds on the OPTA in an asymmetric Gaussian sensor network with deterministic fading [3], [5], we provide a condition for the optimality of analog transmission. Then, we obtain an optimal power allocation scheme for analog transmission in the sense of achieving the optimal LCP-distortion tradeoff. The resulting minimum distortion formula slightly generalizes the result of [6] from a sum-power constraint to an LCP constraint. In addition, we provide a sufficient condition for the optimality of uncoded analog transmission under an LCP constraint.

The remainder of this paper is organized as follows. In Section II, we present the system model and problem statement. Section III briefly reviews known lower and upper bounds on the OPTA in an asymmetric Gaussian sensor network with deterministic fading and provides optimality conditions for analog transmission. In Section IV, we obtain an optimal power allocation under a sum-power (and in general, under an LCP) constraint for analog transmission and provide a sufficient condition for the optimality of analog transmission under an LCP constraint. Conclusions are presented in Section V.

\section{Problem Statement}

We consider a simple Gaussian sensor network, illustrated in Fig. 1, where a team of $L$ sensors observe independent 
noisy versions of the memoryless Gaussian source $\{X[k]\}_{k=1}^{n}$, represented by the sequences $\left\{Y_{i}[k]\right\}_{k=1}^{n}$ for $i=1,2, \ldots, L$. The underlying source $\{X[k]\}_{k=1}^{n}$ is a sequence of independent and identically distributed (i.i.d.) real-valued Gaussian random variables of mean zero and variance $\sigma_{X}^{2}$. For each observation time $k=1,2,3, \ldots$,

$$
Y_{i}[k]=a_{i} X[k]+V_{i}[k], \quad i=1, \ldots, L
$$

where $a_{i} \geq 0$ are fixed and known constants, $\left\{V_{i}[k]\right\}_{k=1}^{n}$ is a sequence of i.i.d. Gaussian random variables of mean zero and variance $N_{i}$ which is independent of $\{X[k]\}_{k=1}^{n}$. We represent the first $n$ instances of $\{X[k]\}_{k>0}$ and $\left\{Y_{i}[k]\right\}_{k>0}$ by the data sequences $X^{n}=(X[1], X[2], \ldots, X[n])$ and $Y_{i}^{n}=$ $\left(Y_{i}[1], Y_{i}[2], \ldots, Y_{i}[n]\right)$, respectively. The correlated sources $Y_{i}$ are not co-located and their observers cannot cooperate to directly exploit their correlation. Instead, the sequences $Y_{i}^{n}$ are separately encoded to $U_{i}^{n}=\varphi_{i}\left(Y_{i}^{n}\right)$ where $\varphi_{i}: \mathbb{R}^{n} \rightarrow \mathbb{R}^{n}, i=$ $1, \ldots, L$ are the encoder functions. Each transmitted sequence $U_{i}^{n}$ is assumed to be average-power limited to $P_{i}$, i.e.,

$$
\frac{1}{n} \sum_{k=1}^{n} E\left[\left|U_{i}[k]\right|^{2}\right] \leq P_{i}, \quad i=1,2, \ldots, L .
$$

The sensors communicate the coded sequences to the decoder through a Gaussian MAC with deterministic (real-valued) fading coefficients $b_{i} \neq 0, i=1, \ldots, L$. The time- $k$ output of the channel is given by

$$
W[k]=\sum_{i=1}^{L} b_{i} U_{i}[k]+Z[k],
$$

where $\{Z[k]\}_{k=1}^{n}$ is an i.i.d. sequence of Gaussian random variables of mean zero and variance $\sigma_{Z}^{2}$. Based on the channel output $W^{n} \in \mathbb{R}^{n}$, the FC forms an estimate $\widehat{X}^{n}$ of the main source $X^{n}$. Fidelity between $X^{n}$ and $\widehat{X}^{n}$ is measured by the average squared error distortion, $\Delta=\frac{1}{n} E\left[\sum_{j=1}^{n}(X[j]-\widehat{X}[j])^{2}\right]$. The reconstructed signal can be described by $\widehat{X}^{n}=\psi\left(W^{n}\right)$, where $\psi: \mathbb{R}^{n} \rightarrow \mathbb{R}^{n}$ is the decoder. Let $\mathcal{F}^{(n)}\left(P_{1}, P_{2}, \ldots, P_{L}\right)$ denote all encoder and decoder functions $\left(\varphi_{1}, \ldots, \varphi_{L}, \psi\right)$ that satisfy (2). For a particular coding scheme $\left(\varphi_{1}, \ldots, \varphi_{L}, \psi\right)$, the performance is determined by the cost vector $\boldsymbol{P}=\left(P_{1}, P_{2}, \ldots, P_{L}\right)$ and the incurred distortion $\Delta$. For any target distortion $D \geq 0$, the power-distortion region $\mathcal{P}(D)$ is defined as the convex closure of the set of all achievable power-distortion pairs $(\boldsymbol{P}, D)$, where a power-distortion pair $(\boldsymbol{P}, D)$ is achievable if for any $\delta>0$, there is an $n_{0}(\delta)$ such that for all $n \geq n_{0}(\delta)$ there exists $\left(\varphi_{1}, \ldots, \varphi_{L}, \psi\right) \in \mathcal{F}^{(n)}\left(P_{1}, P_{2}, \ldots, P_{L}\right)$ with distortion $\Delta \leq D+\delta$. Our aim is to derive optimality conditions for analog transmission in the sense of achieving the OPTA, which is defined for a fixed $\boldsymbol{P}$ as

$$
D_{\text {min }}(\boldsymbol{P})=\inf \{D \mid(\boldsymbol{P}, D) \in \mathcal{P}(D)\} .
$$

In addition, we want to to minimize the MSE distortion given a linear combination of powers (LCP) constraint, i.e.,

$$
\left\{\begin{array}{l}
\text { minimize } \quad D(\boldsymbol{P}) \\
\text { subject to } \quad \sum_{i=1}^{L} \beta_{i} P_{i} \leq \Omega
\end{array}\right.
$$

where $D(\boldsymbol{P})$ is the distortion of the analog transmission scheme using power allocation $\boldsymbol{P}=\left(P_{1}, \ldots, P_{L}\right)$ and $\beta_{i} \geq 0, i=$ $1, \ldots, L$. This new form of constraint is a generalization of the sum-power constraint which explicitly allows to introduce weight coefficients for the potentially differing costs of supplying power to individual sensors.

\section{PRELIMINARIES: OPTA BOUNDS}

In this section, we first review lower and upper bounds on the OPTA in an asymmetric Gaussian sensor network with deterministic fading [3], [5], [7]. The lower bound is based on analyzing a remote source coding scenario where the sensor observations are processed by one common encoder, and then applying a technique based on the notion of the maximum correlation coefficient (see, e.g., [13]). The upper bound is based on analyzing the analog transmission.

\section{A. Lower Bound}

Lemma 1: ([3], [5]) A lower bound on the OPTA in an asymmetric Gaussian sensor network with deterministic fading is given by

$$
D_{m i n} \geq D_{l}=D_{0}^{*}\left(1+\frac{\sigma_{X}^{2} \sigma_{Z}^{2} \sum_{i=1}^{L} \frac{a_{i}^{2}}{N_{i}}}{\sigma_{Z}^{2}+\Omega_{r}}\right)
$$

where

$$
D_{0}^{*}=\left(\frac{1}{\sigma_{X}^{2}}+\sum_{i=1}^{L} \frac{a_{i}^{2}}{N_{i}}\right)^{-1}
$$

and

$$
\Omega_{r}=\sum_{i=1}^{L} \frac{P_{i} N_{i} b_{i}^{2}}{a_{i}^{2} \sigma_{X}^{2}+N_{i}}+\left(\sum_{i=1}^{L} a_{i} b_{i} \sqrt{\frac{P_{i} \sigma_{X}^{2}}{a_{i}^{2} \sigma_{X}^{2}+N_{i}}}\right)^{2} .
$$

\section{B. Upper Bound: Analyzing Analog Transmission}

By analyzing the analog transmission in our Gaussian sensor network, an upper bound on OPTA can be obtained [7]. In this approach, each sensor transmits its observation scaled to its power constraint, i.e., $U_{i}[k]=\sqrt{\frac{P_{i}}{\sigma_{X}^{2}+N_{i}}} Y_{i}[k]$. The received signal at the $\mathrm{FC}$ is then given by

$$
W[k]=\sum_{i=1}^{L}\left\{\sqrt{\frac{P_{i}}{\sigma_{X}^{2}+N_{i}}} b_{i}\left(a_{i} X[k]+V_{i}[k]\right)\right\}+Z[k] .
$$

Since the encoding is memoryless, the optimal estimator is the minimum mean squared error estimator. By evaluating the resulting MSE distortion, an upper bound on the minimum achievable distortion can be obtained; this is summarized in the next lemma.

Lemma 2: ([3], [5], [7]) An upper bound on the OPTA in a Gaussian sensor network with deterministic fading can be expressed as

$$
D_{\text {min }} \leq D_{\text {uncoded }}=\left(\frac{\sigma_{X}^{2}}{\sigma_{Z}^{2}+\Omega_{r}}\right)\left(\sigma_{Z}^{2}+\sum_{i=1}^{L} \frac{P_{i} N_{i} b_{i}^{2}}{a_{i}^{2} \sigma_{X}^{2}+N_{i}}\right),
$$

where $\Omega_{r}$ is given in (8). 
In the symmetric case, where $N_{1}=N_{2}=\cdots=N_{L}=N$ and $P_{1}=P_{2}=\cdots=P_{L}=P$, and when $a_{1}=a_{2}=\cdots=$ $a_{L}=1$, the lower bound (6) and the upper bound (9) coincide if $b_{1}=b_{2}=\cdots=b_{L}=b$. Hence, we obtain the OPTA for the symmetric Gaussian sensor network under deterministic and identical fading. This result was recently established by Gastpar in [3], [7] with $b_{1}=b_{2}=\cdots=b_{L}=1$.

\section{Optimality Conditions}

Corollary 1: The lower bound (6) and the upper bound (9) coincide if and only if

$$
\frac{b_{1}}{a_{1}} N_{1} \sqrt{\frac{P_{1}}{a_{1}^{2} \sigma_{X}^{2}+N_{1}}}=\cdots=\frac{b_{L}}{a_{L}} N_{L} \sqrt{\frac{P_{L}}{a_{L}^{2} \sigma_{X}^{2}+N_{L}}} .
$$

Proof: We first rewrite the lower bound in (6) as

$$
D_{l}=\frac{\sigma_{X}^{2} \sigma_{Z}^{2}+D_{0}^{*} \sum_{i=1}^{L} \frac{P_{i} N_{i} b_{i}^{2}}{a_{i}^{2} \sigma_{X}^{2}+N_{i}}+D_{0}^{*}\left(\sum_{i=1}^{L} a_{i} b_{i} \sqrt{\frac{P_{i} \sigma_{X}^{2}}{a_{i}^{2} \sigma_{X}^{2}+N_{i}}}\right)^{2}}{\sigma_{Z}^{2}+\sum_{i=1}^{L} \frac{P_{i} N_{i} b_{i}^{2}}{a_{i}^{2} \sigma_{X}^{2}+N_{i}}+\left(\sum_{i=1}^{L} a_{i} b_{i} \sqrt{\frac{P_{i} \sigma_{X}^{2}}{a_{i}^{2} \sigma_{X}^{2}+N_{i}}}\right)^{2}} .
$$

Comparing (11) with the upper bound in (9) reveals that the lower bound and the upper bound coincide if and only if

$\left(\sigma_{X}^{2}-D_{0}^{*}\right) \sum_{i=1}^{L} \frac{P_{i} N_{i} b_{i}^{2}}{a_{i}^{2} \sigma_{X}^{2}+N_{i}}=D_{0}^{*} \sigma_{X}^{2}\left(\sum_{i=1}^{L} a_{i} b_{i} \sqrt{\frac{P_{i}}{a_{i}^{2} \sigma_{X}^{2}+N_{i}}}\right)^{2}$

From the definition of $D_{0}^{*}$ we have $\frac{\sigma_{X}^{2}-D_{0}^{*}}{\sigma_{X}^{2} D_{0}^{*}}=\sum_{i=1}^{L} \frac{a_{i}^{2}}{N_{i}}$. As a result, the equality condition in (12) is equivalent to

$$
\left(\sum_{i=1}^{L} \frac{a_{i}^{2}}{N_{i}}\right) \sum_{i=1}^{L} \frac{P_{i} N_{i} b_{i}^{2}}{a_{i}^{2} \sigma_{X}^{2}+N_{i}}=\left(\sum_{i=1}^{L} a_{i} b_{i} \sqrt{\frac{P_{i}}{a_{i}^{2} \sigma_{X}^{2}+N_{i}}}\right)^{2} .
$$

Setting $g_{i}=\frac{b_{i}}{a_{i}} N_{i} \sqrt{\frac{P_{i}}{a_{i}^{2} \sigma_{X}^{2}+N_{i}}}$, we rewrite (13) as

$$
\left(\sum_{i=1}^{L} \frac{a_{i}^{2}}{N_{i}}\right) \sum_{i=1}^{L} \frac{a_{i}^{2} g_{i}^{2}}{N_{i}}=\left(\sum_{i=1}^{L} \frac{a_{i}^{2} g_{i}}{N_{i}}\right)^{2} .
$$

This condition can be rearranged as

$$
\sum_{i=1}^{L} \frac{a_{i}^{2} g_{i}^{2}}{N_{i}}\left(\sum_{j \neq i}^{L} \frac{a_{j}^{2}}{N_{j}}\right)=2 \sum_{i=1}^{L} \sum_{j>i} \frac{a_{i}^{2} a_{j}^{2} g_{i} g_{j}}{N_{i} N_{j}}
$$

Comparing the coefficients of $\frac{1}{N_{i} N_{j}}$ on both sides of (15) shows that the equality holds if and only if $g_{i}^{2}+g_{j}^{2}=2 g_{i} g_{j}$; this is equivalent to $g_{i}=g_{j}$ which is our condition in (10).

Hence, for a given set of powers, if (10) is satisfied then the distortion achieved by the analog transmission is the smallest possible achievable distortion. Note that (10) is both necessary and sufficient for the upper and lower bounds to coincide, but it is only a sufficient condition for the optimality of analog transmission.

\section{Optimal Power Allocation for Analog TRANSMISSION UNDER AN LCP CONSTRAINT}

We consider an optimal power allocation for the analog transmission in order to minimize the MSE distortion under an
LCP constraint, i.e.,

$$
\left\{\begin{array}{l}
\text { minimize } \quad D(\boldsymbol{P}) \\
\text { subject to } \quad \sum_{i=1}^{L} \beta_{i} P_{i} \leq \Omega
\end{array}\right.
$$

where $D(\boldsymbol{P})$ is the distortion of the analog transmission scheme using power allocation $\boldsymbol{P}=\left(P_{1}, \ldots, P_{L}\right)$ and $\beta_{i} \geq 0, i=$ $1, \ldots, L$. In [6], by applying the Lagrange multiplier method, it was shown that the optimal distortion sum-power tradeoff $\left(D_{\text {uncoded }}, \sum_{i=1}^{L} P_{i}\right)$ for the analog scheme can be expressed as follows:

$$
D_{\text {uncoded }}(\bar{P})=\left(\frac{1}{\sigma_{X}^{2}}+\sum_{j=1}^{L} \frac{a_{j}^{2}}{N_{j}+\frac{\sigma_{Z}^{2}}{\bar{P} b_{j}^{2}}\left(a_{j}^{2} \sigma_{X}^{2}+N_{j}\right)}\right)^{-1}
$$

where $\bar{P}=\sum_{i=1}^{L} P_{i}$. This tradeoff is also given in [7, Eq. (6)] for the case of equal variance observation noises, $a_{j}=1$ for all $j$ and equal channel gains.

Unfortunately, [6] does not seem to provide a closed form expression for the power allocation achieving the minimum distortion for a given sum-power $\bar{P}$. Since we want to compare lower and upper bounds on OPTA under an LCP constraint (which subsumes the sum-power constraint), we first need to provide closed form expressions of the optimal power allocation for the LCP case. The proof of the next lemma, which we omit, is based on an application of the Lagrange multiplier method. Note that as a special case, the lemma also yields the formula derived in [6] for an optimal power allocation achieving the minimum distortion.

Lemma 3: (Optimal Power Allocation for Analog Transmission under an LCP Constraint) An optimal power allocation for the constraint $\Omega=\sum_{i=1}^{L} \beta_{i} P_{i}$ is given by

$$
P_{i}=\lambda_{i} \Omega, \quad i=1, \ldots, L
$$

where

$$
\lambda_{i}=\frac{a_{i}^{2}\left(a_{i}^{2} \sigma_{X}^{2}+N_{i}\right)}{\alpha b_{i}^{2}\left(\Omega N_{i}+\sigma_{Z}^{2} \beta_{i}\left(\frac{a_{i}^{2} \sigma_{X}^{2}+N_{i}}{b_{i}^{2}}\right)\right)^{2}},
$$

and

$$
\alpha=\sum_{j=1}^{L} \frac{\beta_{j} a_{j}^{2}\left(a_{j}^{2} \sigma_{X}^{2}+N_{j}\right)}{b_{j}^{2}\left(\Omega N_{j}+\sigma_{Z}^{2} \beta_{j}\left(\frac{a_{j}^{2} \sigma_{X}^{2}+N_{j}}{b_{j}^{2}}\right)\right)^{2}} .
$$

Substituting the optimal $P_{i}$ given by (18), into (9) yields an upper bound on OPTA under the LCP constraint:

$$
D_{\text {uncoded }}(\Omega)=\left(\frac{1}{\sigma_{X}^{2}}+\sum_{j=1}^{L} \frac{a_{j}^{2}}{N_{j}+\frac{\sigma_{Z}^{2} \beta_{j}}{\Omega b_{j}^{2}}\left(a_{j}^{2} \sigma_{X}^{2}+N_{j}\right)}\right)^{-1} .
$$

Corollary 2: (Analog Transmission Optimality Conditions under an LCP Constraint) In an asymmetric Gaussian sensor network with deterministic fading, analog transmission scheme with the optimal power allocation given in (18) is optimal in the sense of achieving the OPTA under an LCP constraint if the 
following conditions hold

$$
\frac{b_{1}}{a_{1}} \sqrt{\frac{N_{1}}{\beta_{1}\left(\sigma_{X}^{2}+\frac{N_{1}}{a_{1}^{2}}\right)}}=\cdots=\frac{b_{L}}{a_{L}} \sqrt{\frac{N_{L}}{\beta_{L}\left(\sigma_{X}^{2}+\frac{N_{L}}{a_{L}^{2}}\right)}} .
$$

Proof: From the proof of Corollary 1, a necessary and sufficient condition for the upper and the lower bounds to coincide is that the equality in (13) holds. Substituting the optimal $P_{i}$ 's we obtain

$$
\begin{aligned}
& \left(\sum_{i=1}^{L} \frac{a_{i}^{2}}{N_{i}}\right) \frac{\Omega}{\alpha} \sum_{i=1}^{L} \frac{a_{i}^{2} N_{i}}{\left(\Omega N_{i}+\sigma_{Z}^{2} \beta_{i}\left(\frac{a_{i}^{2} \sigma_{X}^{2}+N_{i}}{b_{i}^{2}}\right)\right)^{2}} \\
= & \left(\sum_{i=1}^{L} a_{i} b_{i} \sqrt{\frac{a_{i}^{2} \Omega}{\alpha b_{i}^{2}\left(\Omega N_{i}+\sigma_{Z}^{2} \beta_{i}\left(\frac{a_{i}^{2} \sigma_{X}^{2}+N_{i}}{b_{i}^{2}}\right)\right)^{2}}}\right)^{2} .
\end{aligned}
$$

Denoting $f_{i}=\frac{N_{i}}{\Omega N_{i}+\sigma_{Z}^{2} \beta_{i}\left(\frac{a_{i}^{2} \sigma_{X}^{2}+N_{i}}{b_{i}^{2}}\right)}$, we can rewrite (21) in terms of the $f_{i}$ 's as follows

$$
\begin{aligned}
\sum_{i=1}^{L} \frac{f_{i}^{2} a_{i}^{2}}{N_{i}}\left(\sum_{j=1}^{L} \frac{a_{j}^{2}}{N_{j}}\right)= \\
\quad \sum_{i=1}^{L} \frac{a_{i}^{4} f_{i}^{2}}{N_{i}^{2}}+2 \sum_{i=1}^{L} \sum_{j>i} \frac{a_{i}^{2} a_{j}^{2} f_{i} f_{j} \operatorname{sgn}\left(b_{i}\right) \operatorname{sgn}\left(b_{j}\right)}{N_{i} N_{j} \operatorname{sgn}\left(a_{i}\right) \operatorname{sgn}\left(a_{j}\right)},
\end{aligned}
$$

where $\operatorname{sgn}(x)=\frac{x}{|x|}$ is the sign of $x$. Subtracting $\sum_{i=1}^{L} \frac{a_{i}^{4} f_{i}^{2}}{N_{i}^{2}}$ from both sides in (22), we get

$$
\sum_{i=1}^{L} \frac{f_{i}^{2} a_{i}^{2}}{N_{i}}\left(\sum_{j \neq i}^{L} \frac{a_{j}^{2}}{N_{j}}\right)=2 \sum_{i=1}^{L} \sum_{j>i} \frac{a_{i}^{2} a_{j}^{2} f_{i} f_{j} \operatorname{sgn}\left(b_{i}\right) \operatorname{sgn}\left(b_{j}\right)}{N_{i} N_{j} \operatorname{sgn}\left(a_{i}\right) \operatorname{sgn}\left(a_{j}\right)} .
$$

Comparing the coefficients of $\frac{1}{N_{i} N_{j}}$ in both sides of equation (23) gives that the equality holds if and only if

$$
f_{i}^{2}+f_{j}^{2}=\frac{2 f_{i} f_{j} \operatorname{sgn}\left(b_{i}\right) \operatorname{sgn}\left(b_{j}\right)}{\operatorname{sgn}\left(a_{i}\right) \operatorname{sgn}\left(a_{j}\right)} .
$$

Since $f_{i} \geq 0$, the equality holds if and only if $f_{i}=f_{j}$ and $\frac{\operatorname{sgn}\left(b_{i}\right) \operatorname{sgn}\left(b_{j}\right)}{\operatorname{sgn}\left(a_{i}\right) \operatorname{sgn}\left(a_{j}\right)}=1$. Solving $f_{i}=f_{j}$ implies that

$$
\frac{\beta_{i}}{N_{i}} \frac{a_{i}^{2} \sigma_{X}^{2}+N_{i}}{b_{i}^{2}}=\frac{\beta_{j}}{N_{j}} \frac{a_{j}^{2} \sigma_{X}^{2}+N_{j}}{b_{j}^{2}} .
$$

Combining (24) with $\operatorname{sgn}\left(b_{i}\right) \operatorname{sgn}\left(b_{j}\right)=\operatorname{sgn}\left(a_{i}\right) \operatorname{sgn}\left(a_{j}\right)$, we obtain (20) which completes the proof.

Example 1: We assume that the source is an i.i.d. zero mean Gaussian process with variance $\sigma_{X}^{2}=80$. We also assume that $L=2, N_{1}=10, N_{2}=20, \sigma_{Z}^{2}=15, a_{1}=a_{2}=1, b_{1}=b_{2}=1$ and set the LCP constraint as $\Omega=\sum_{i=1}^{L} \beta_{i} P_{i}=8$. Fig. 2 shows the mean-squared estimation distortion as a function of the cost weight $\beta_{1}$ where we assume that $\beta_{2}=k_{\beta} \beta_{1}$. The figure is parametrized by three different values of $k_{\beta}$. When the cost weight $\beta_{i}$ is small, the system increases power allocation $P_{i}$ to achieve lower values of distortion. Larger values of the cost weight will result in larger achievable distortions.

In Fig. 3, the optimal percentage of the total power assigned to

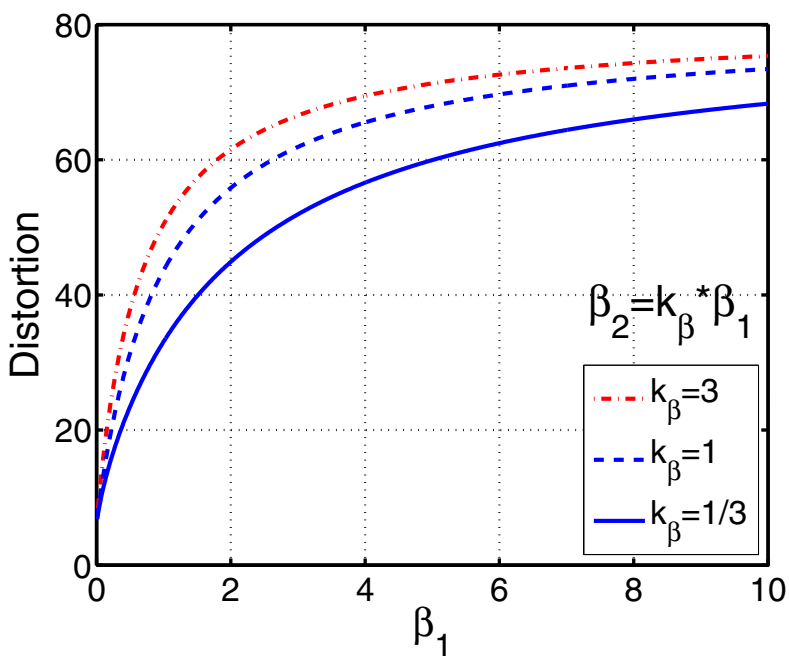

Fig. 2. Optimal achievable distortion v.s. the cost weight $\beta_{1}$, parametrized by the value of $k_{\beta}$ where $k_{\beta}=\frac{\beta_{2}}{\beta_{1}}$. We assume that $N_{1}=10, N_{2}=20$, $\sigma_{X}^{2}=80, \sigma_{Z}^{2}=15, a_{1}=a_{2}=1, b_{1}=b_{2}=1$ and the LCP constraint, $\Omega=\sum_{i=1}^{L} \beta_{i} P_{i}=8$.

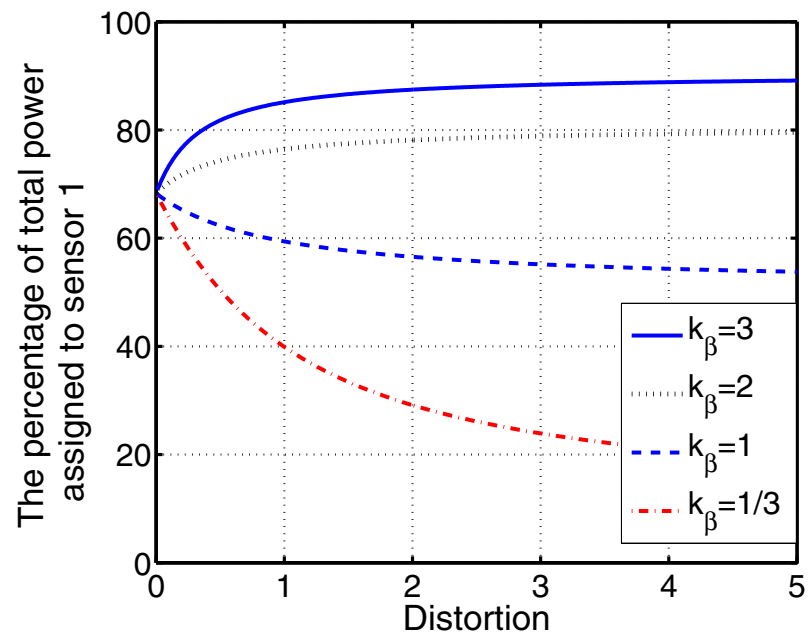

Fig. 3. The percentage of the total power assigned to sensor 1 in the sensor network of $L=2$ sensors. We assume that $\sigma_{X}^{2}=10, N_{1}=1, N_{2}=1.5$, $\sigma_{Z}^{2}=1, a_{1}=a_{2}=1, b_{1}=b_{2}=1$ and the LCP constraint equals to 8 .

sensor 1 for the case of $L=2$ sensors is illustrated. We observe that the optimal power allocation depends on the desired degree of fidelity, noise variances and the ratio of cost weights. When it is expensive to transmit, lower power is assigned to the sensor with a higher cost element. When all the cost elements are equal, more power is assigned to the sensor with lower noise level.

Note that by substituting the optimal $P_{i}$ (under the sum-power constraint and $a_{i}=1$ for $\left.i=1, \ldots, L\right)$ in the lower bound of distortion in (6), we are able to compare the lower and upper bounds numerically. The lower bound can be expressed as

$$
D_{l}(\bar{P})=D_{0}^{*}\left(1+\frac{\alpha \bar{P} \sigma_{Z}^{2} D_{\text {uncoded }}(\bar{P}) \sum_{i=1}^{L} \frac{1}{N_{i}}}{\frac{1}{D_{\text {uncoded }}(\bar{P})}-\frac{1}{\sigma_{X}^{2}}}\right) .
$$

First, we consider an asymmetric Gaussian sensor network with 


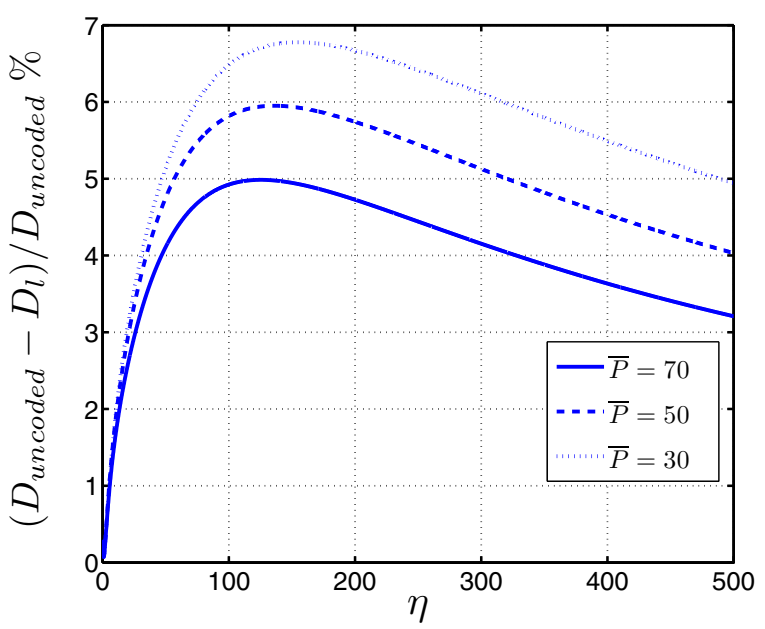

Fig. 4. Percentage of the relative distortion gap versus the value of $\eta$ where $N_{i}=N_{i-1}+\eta$ (for $\left.i=2, \ldots, L\right)$ and $N_{1}=20$. The figure is parametrized by the total power $\bar{P}=P_{1}+\cdots+P_{L}$. We assume that $L=20, \sigma_{X}^{2}=100, \sigma_{Z}^{2}=$ 20. The fading coefficients are as follows: $b_{i}= \begin{cases}1 & i=1, \ldots, 8 \\ 0.9 & i=9, \ldots, 16 \\ 0.8 & i=17, \ldots, 20\end{cases}$

$L=20$ sensors, where $N_{i}=N_{i-1}+\eta$ (for $i=2, \ldots, L$ ) and $N_{1}=20$. The fading coefficients of the channels are as follows:

$$
b_{i}= \begin{cases}1 & i=1, \ldots, 8 \\ 0.9 & i=9, \ldots, 16 \\ 0.8 & i=17, \ldots, 20\end{cases}
$$

We plot the percentage of the relative distortion gap (i.e., $\frac{D_{\text {uncoded }}-D_{l}}{D_{\text {uncoded }}} \%$ ) versus the value of $\eta$, parametrized by the sumpower $P$, see Fig. 4 . The gap is less than $7 \%$ for all values of $\eta$ as well as all three values of $\bar{P}$. The gap also decreases with increasing $\bar{P}$.

Fig. 5 shows the relative distortion gap versus the total number of sensors, $L$, under the sum-power constraint $\bar{P}=50$. For this figure, we assume semi-symmetric sensor observations, i.e., $N_{i}=N_{i-1}$ for $i=2, \ldots,\lfloor L / 2\rfloor$ and $N_{i}=N_{i-1}+\eta$ for $i=\lfloor L / 2\rfloor+1, \ldots, L$ where $N_{1}=10$ and $\eta=5$. The fading coefficients are $b_{i}=1$ for $i=2, \ldots,\lfloor L / 2\rfloor$ and $b_{i}=0.9$ for $i=\lfloor L / 2\rfloor+1, \ldots, L$. We observe that analog transmission can achieve a near optimal performance under a sum-power constraint.

\section{CONCLUSIONS}

In this paper, we considered a distributed sensor network where $L$ noisy observations of a memoryless Gaussian source are analog transmitted through a Gaussian MAC to a decoder. The decoder wants to reconstruct the main source with an average distortion $D$ at the smallest possible power consumption in the communication link. For a symmetric sensor network with no fading, it is known that analog transmission achieves the OPTA [3], [7], [8]. Here we provided conditions for analog transmission in an asymmetric Gaussian sensor network with deterministic fading to achieve the OPTA. We also obtained an optimal power allocation to minimize the MSE distortion for a given linear combination of powers (LCP) constraint. We used the optimal power allocations to derive a condition for the

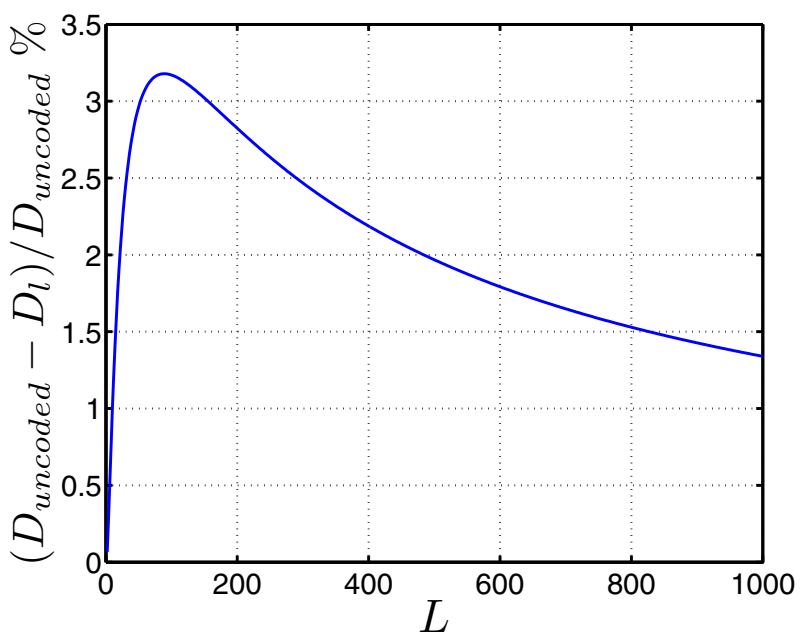

Fig. 5. Percentage of the relative distortion gap versus the total number of sensors, $L$, under the sum-power constraint. We assume that $N_{i}=N_{i-1}$ for $i=2, \ldots,\lfloor L / 2\rfloor$ and $N_{i}=N_{i-1}+\eta$ for $i=\lfloor L / 2\rfloor+1, \ldots, L$. The fading coefficients are $b_{i}=1$ for $i=2, \ldots,\lfloor L / 2\rfloor$ and $b_{i}=0.9$ for $i=$ $\lfloor L / 2\rfloor+1, \ldots, L$. Other parameters are $N_{1}=10, \eta=5, \bar{P}=50, \sigma_{X}^{2}=100$, $\sigma_{Z}^{2}=20$.

analog transmission scheme to achieve the OPTA under an LCP constraint.

\section{REFERENCES}

[1] T. J. Goblick, "Theoretical limitations on the transmission of data from analog sources," IEEE Trans. Inf. Theory, vol. 11, no. 4, pp. 558-567, Oct. 1965.

[2] M. Gastpar, B. Rimoldi, and M. Vetterli, "To code, or not to code: Lossy source channel communication revisited," IEEE Trans. Inf. Theory, vol. 49, no. 5, pp. 1147-1158, May 2003.

[3] M. Gastpar, "Uncoded transmission is exactly optimal for a simple Gaussian sensor network," IEEE Trans. Inf. Theory, vol. 54, no. 11, pp. 5247-5251, Nov. 2008.

[4] A. Lapidoth and S. Tinguely, "Sending a bi-variate Gaussian source over a Gaussian MAC," in Proc. IEEE Int. Symp. Inf. Theory (ISIT), Seattle, Washington, Jul. 2006, pp. 2124-2128.

[5] H. Behroozi, F. Alajaji, and T. Linder, "On the optimal power-distortion region for asymmetric Gaussian sensor networks with fading," in Proc. IEEE Int. Symp. Inf. Theory (ISIT), Toronto, ON, Canada, Jul. 2008.

[6] J. Xiao, Z.-Q. Luo, S. Cui, and A. Goldsmith, "Power-efficient analog forwarding transmission in an inhomogeneous Gaussian sensor network," in Proc. 2005 IEEE 6th Workshop on Signal Processing Advances in Wireless Communications, Jun. 2005, pp. 121-125.

[7] M. Gastpar, "Uncoded transmission is exactly optimal for a simple Gaussian sensor network," in Proc. 2007 Information Theory and Applications Workshop, San Diego, CA, USA, Jan. 2007.

[8] H. Behroozi and M. R. Soleymani, "On the optimal power-distortion tradeoff in asymmetric Gaussian sensor network," IEEE Trans. Commun., Accepted, Sept. 2008.

[9] R. Rajesh and V. Sharma, "Source-channel coding for Gaussian sources over a Gaussian multiple access channel," in Proc. the 45th Annual Allerton Conference on Communications, Control and Computing, Urbana, Illinois, Sep. 2007.

[10] C. E. Shannon, "A mathematical theory of communication," Bell Systems Technical Journal, vol. 27, pp. 379-423, pp. 623-656, 1948.

[11] M. Gastpar and M. Vetterli, "Power, spatio-temporal bandwidth, and distortion in large sensor networks," IEEE J. Sel. Areas Commun., vol. 23, no. 4, pp. 745-754, Apr. 2005.

[12] S. Bross, A. Lapidoth, and S. Tinguely, "Broadcasting correlated Gaussians," in Proc. IEEE Int. Symp. Inf. Theory (ISIT), Toronto, ON, Canada, Jul. 2008.

[13] H. S. Witsenhausen, "On sequences of pairs of dependent random variables," SIAM J. Appl. Math., vol. 28, no. 1, pp. 100-113, Jan. 1975. 